\title{
ON MAXIMAL GROUPS OF ISOMETRIES
}

\author{
LUDVIK JANOS
}

\begin{abstract}
The purpose of this note is to introduce the concept of "Optimal Metrization" for metrizable topological spaces. Let $X$ be such a space, $\rho$ a metric on $X$ and $K(\rho)$ the group of all those homeomorphisms of $X$ onto itself which preserve $\rho$. The metric $\rho$ is said to be "optimal" provided there is no $\rho^{*}$ with $K\left(\rho^{*}\right)$ properly containing $K(\rho)$. A space having at least one optimal metric is called "optimally metrizable." Examples of spaces which are and which are not optimally metrizable are given; it is shown that the real line $R$ is, and that the usual metric is optimal.
\end{abstract}

1. Introduction and notation. Let $X$ be a metrizable topological space. We denote by $G(X)$ the group of all homeomorphisms of $X$ onto itself and by $M(X)$ the set of all metrics on $X$ compatible with the topology of $X$. We observe that with each $\rho \in M(X)$ there is associated the subgroup $K(\rho) \subseteq G(X)$ (group of all isometries for $\rho$ ) defined by $K(\rho)=\{h \mid h \in G(X)$ and $\rho(x, y)=\rho(h(x), h(y))$ for all $x, y \in X\}$. The basic idea motivating our investigations is the classification of a metric $\rho \in M(X)$ according to the size of the corresponding group $K(\rho)$.

Convention. In this paper the set-theoretical inclusion is denoted by $\supseteq$, reserving $\supset$ for the proper inclusion.

Definition 1.1. A metric $\rho \in M(X)$ is said to be optimal iff there is no $\rho^{*} \in M(X)$ with $K\left(\rho^{*}\right) \supset K(\rho)$. A space $X$ is said to be optimally metrizable iff there is at least one optimal metric in $M(X)$.

Denoting by $L(X)$ the lattice of all subgroups of $G(X)$ we have the mapping $K: M(X) \rightarrow L(X)$ defined by $K(\rho) \in L(X)$ for $\rho \in M(X)$.

Definition 1.2. The image of $M(X)$ under $K$ is the subset $P(X)$ $\subseteq L(X)$ partially ordered by inclusion. Its elements are groups of isometries and its maximal element (if it exists) is called a maximal group of isometry.

It is obvious that $X$ is optimally metrizable if and only if $P(X)$ has a maximal element.

If $A \in L(X)$ and $h \in G(X)$ we denote by $(A, h)$ the subgroup generated by $A$ and $h$.

REMARK 1.1. It is obvious that a space $X$ is not optimally metrizable iff to each $A \in P(X)$ there exists $h \in G(X)$ such that $h \notin A$ and $(A, h) \in P(X)$.

Received by the editors May 3, 1970.

AMS 1969 subject classifications. Primary 5480; Secondary 2240.

Key words and phrases. Group of isometries, optimal metric, optimally metrizable. 
2. General properties of the set $P(X)$. We observe that if $\rho \in M(X)$ then for each $g \in G(X)$ the function $g \rho$ defined by $g \rho(x, y)=\rho(g(x), g(y))$ for all $x, y \in X$ is again a metric $\in M(X)$; thus $G(X)$ acts on $M(X)$ in this natural way.

THEOREM 2.1. For each $\rho \in M(X)$ and $g \in G(X)$ we have $K(g \rho)$ $=g^{-1} K(\rho) g$. Thus $P(X)$ contains with each $A \in P(X)$ all its conjugates $g^{-1} A g \in P(X)$.

Proof. By straightforward verification.

COROLlaRY. If $\rho \in M(X)$ is optimal then go is also optimal for every $g \in G(X)$.

In the case $X$ is compact we topologize $G(X)$ by the uniform convergence topology and it is a well-known fact (see for example [1] and [2]) that any compact subgroup $K$ of $G(X)$ lies inside $K(\rho)$ for some $\rho$. Hence we obtain this obvious statement.

Theorem 2.2. If $X$ is compact then $X$ is optimally metrizable iff $G(X)$ has a maximal compact subgroup.

3. Optimal metrization property of some well-known spaces. We first observe that any set $X$ with the discrete topology is optimally metrizable and the optimal metric $\rho$ is the most trivial one defined by $\rho(x, y)=1$ for $x \neq y$. In this case we have $K(\rho)=G(X)$ and $P(X)$ $=L(X)$. On the other hand we now show that the one-point compactification $N^{*}$ of the set of positive integers $N$ has not this property. Under $N^{*}$ we understand the set $N \cup\{\infty\}$ metrized for example by: $\rho(n, m)=|1 / n-1 / m|$ for $n, m \in N$ and $\rho(n, \infty)=1 / n$ for $n \in N$.

THEOREM 3.1. The space $N^{*}$ is not optimally metrizable.

Proof. Suppose that $K$ were a maximal compact subgroup of $G\left(N^{*}\right)$. Any orbit $K(n)=\{g(n) \mid g \in K\}$ is compact; so if it were infinite, then it would include $\infty$. But no member of $G\left(N^{*}\right)$ moves $\infty$, so $K(n)$ is finite. Thus there are $n, m \in N$ with disjoint orbits $K(n), K(m)$. Taking for $h$ the simple transposition of $m$ and $n$, we observe that the action of $(K, h)$ differs from that of $K$ only on the finite set $K(n) \cup K(m)$, and $(K, h)$ is therefore again compact, which is impossible. Hence, $N^{*}$ is not optimally metrizable.

THEOREM 3.2. The compact interval $[a, b]$ is optimally metrizable and the usual metric $|y-x|$ is optimal.

Proof. We prove this showing that the group $K=\{e, r\}$ consisting of the identity $e$ and the reflexion $r(r(x)=a+b-x$ for $x \in[a, b])$ is maximal compact in $G([a, b])$. If this were not the case then there 
would exist a larger compact group $K^{\prime} \supset K$. If $h \in K^{\prime} \backslash K$ we may assume $h$ is increasing since $r \cdot h$ also belongs to $K^{\prime} \backslash K$. Since $h \neq e$ there is $p \in[a, b]$ such that $h(p) \neq p$, and we know that there is an interval $[c, d] \subseteq[a, b]$ with $p$ in the interior and the only points fixed by $h$ are $c$ and $d$. Now $\left\{h^{n} \mid n \geqq 1\right\}$ has a limit element $g$ in $K^{\prime}$, so $g(c)$, $g(p), g(d)$ are limit points of $\left\{h^{n}(c)\right\},\left\{h^{n}(p)\right\},\left\{h^{n}(d)\right\}$ respectively. Thus $g(c)=c, g(d)=d$ and $g(p)=c$ or $d$ which is impossible. Hence $K^{\prime}=K=\{e, r\}=K(\rho)$ where $\rho(x, y)=|y-x|$ showing that this metric is optimal.

\section{THEOREM 3.3. The circle $S_{1}$ is optimally metrizable.}

Proof. Representing the circle $S_{1}$ in the form $S_{1}=\left\{e^{i \pi x} \mid x \in[-1,1]\right\}$ we shall prove that the group $G \subseteq G\left(S_{1}\right)$ consisting of all rotations $e^{i \pi x} \rightarrow e^{i \pi x(x+a)}$ and the reflexion $e^{i \pi x} \rightarrow e^{-i \pi x}$ is a maximal compact subgroup. To this end we observe that the set $G\left(S_{1} ;-1\right) \subseteq G\left(S_{1}\right)$ of all those elements of $G\left(S_{1}\right)$ which leave invariant the point $-1=e^{-i \pi}$ is a closed subgroup of $G\left(S_{1}\right)$ which is homeomorphic and isomorphic to $G[-1,1]$. If $G^{*}$ were a compact group containing $G$, then according to Theorem $3.2 G^{*} \cap G\left(S_{1} ;-1\right)=G \cap G\left(S_{1} ;-1\right)$. Now if $g$ belongs to $G^{*}$, then we can find a rotation $f$ such that $f\left(e^{-i \pi}\right)=g\left(e^{-i \pi}\right)$. Thus $h=f^{-1} g$ belongs to $G^{*} \cap G\left(S_{1} ;-1\right), h$ belongs to $G, g=f h$ belong to $G$ and $G^{*}=G$ which proves our assertion.

TheоReм 3.4. The real line $R$ is optimally metrizable and the usual metric $|y-x|$ is optimal.

PROof. The metric $|y-x|$ is preserved by the group of all translations and reflexions. Denoting this group by $K$, assume that there is $\rho \in M(R)$ with $K(\rho) \supset K$. Let $f \in K(\rho) \backslash K$. Without loss of generality we may assume $f$ increasing and having at least one fixed point since otherwise we would apply on $f$ suitable operations in $K$. Let $F$ be the set of all fixed points of $f$. From $F \neq \varnothing$ we know that $R \backslash F$ has a connected component $C$ that is an interval with at least one endpoint $a$. If we choose $b$ in $C$, then $\left\{f^{n}(b)\right\}$ or $\left\{f^{-n}(b)\right\}$ will approach $a$, but $\rho\left(f^{n+1}(b), f^{n}(b)\right)=\rho(f(b), b)=\rho\left(f^{-n-1}(b), f^{-n}(b)\right)$ will not approach 0 , which is impossible. Thus $K$ is the maximal group of isometry corresponding to the metric $|y-x|$ which completes our proof.

\section{REFERENCES}

1. S. Eilenberg, Sur les groupes compacts d'homéomorphies, Fund. Math. 28 (1937), 75-80.

2. M. T. Wechsler, Homeomorphism groups of certain topologicai spaces, Ann. of Math. (2) 62 (1955), 360-373. MR 17, 287.

University of Florida, Gainesville, Florida 32601 